\title{
Challenges in Determining Structure of Supported Subnano Metal Clusters
}

\author{
Jingyue (Jimmy) Liu ${ }^{1 *}$ \\ 1. Department of Physics, Arizona State University, Tempe, Arizona, USA. \\ * Corresponding author: Jingyue.liu@asu.edu
}

Subnano metal clusters exhibit unique optical, catalytic, electronic and magnetic properties that are not observed in their corresponding bulk counterparts. Such distinct behaviors of metal clusters can be attributed to many factors including quantum confinement, high surface-to-bulk atom ratio, and their unique size- and configuration-dependent electronic shell structures. Supported subnano clusters are of particular interest in catalysis since small changes in atomicity, composition, and/or even their geometric configuration can significantly influence their catalytic activity and selectivity [1-2]. Supported metal clusters at the single atom limit have yielded excellent performance for catalytic transformation of a variety of important molecules [3]. Strong metal-support interactions become dominant for supported subnano clusters or single atoms [3-5]. A major obstacle to gaining a deeper understanding of catalysis by supported clusters is the lack of information on their geometric configurations and electronic structures.

Aberration-corrected electron microscopy, especially aberration-corrected STEM, has made significant contributions to characterizing supported metal particles and single atoms [4-5]. To accurately determine the structure of supported subnano metal clusters in practical supported metal catalysts, however, has been a grand challenge. Electron-beam induced effects are the main culprits for achieving the elusive goal of studying structure-performance relationships of supported subnano metal clusters. Although electron microscopists have been enjoying the "dancing" atoms and clusters inside an electron microscope for decades it is a non-trivial task to keep the atoms and clusters stable and thus provide useful information on the "intact structure" of supported metal atoms and clusters. Electron-bean induced effects have been extensively studied by electron microscopists [6]. Both knock-on and radiolysis processes, and in some cases electrostatic charging effects, can displace surface adatoms and modify cluster configurations. Because of the radiolysis processes lower accelerating voltages may not always help. Furthermore, lowenergy electrons cannot penetrate thicker samples without suffering the loss of image resolution even if both geometric and chromatic aberrations are corrected.

Many microscope parameters (accelerating voltage, total dose and dose rate, pixel dwell time, detector choice, etc.) need to be optimized in order to obtain relatively useful information on the structure of supported metal clusters. Sample related parameters, such as the nature of the metal clusters, the support surfaces, and the anchoring strength of the metal clusters to the support surfaces, determine the choice of the microscope parameters. Figure 1 shows a HAADF image of $2 \mathrm{D} \mathrm{Co}_{3} \mathrm{O}_{4}$ nanosheets supported $\mathrm{Pt}$ atoms, subnano clusters, and small particles. Both the Pt single atoms and subnano clusters moved significantly during the observation process. Although the bulk structures of the small Pt particles (indicated by the letter 4) were relatively stable the surface atoms on these small Pt particles, which are crucial to catalysis, constantly changed under the electron beam irradiation. In this particular case, it was impossible to obtain useful information on the specific geometric configurations of the subnano Pt clusters, even with the use of low-dose electron probe. When metal clusters anchor strongly onto the support surfaces, however, they may become stable under low-dose electron beam irradiation. Figure 2a shows a HAADF image of a subnano Ir cluster that was epitaxially anchored onto the surface of a $\mathrm{ZnO}$ nanowire, clearly revealing the crystal structure and the atomic arrangement of the Ir atoms. Even after prolonged electron beam 
irradiation (5 min) the subano Ir cluster maintained its structure and configuration (Fig. 2b). When the support material is sensitive to electron beam and the anchoring of the metal atoms and clusters is weak then the electron-beam induced effects become severe even if low-dose electron beam and ultra-fast scan rate were used. Figure $2 \mathrm{c}$ shows a HAADF image of 2D MoS 2 supported Pt atoms and subnano clusters. After only one scan, the Pt atoms moved drastically and the Pt subnano clusters completely changed their configurations and even atomicity (Fig. 2d). The edges of the $\mathrm{MoS}_{2}$ support surfaces were modified as well. Strategies to alleviate electron-beam induced effects will be discussed [7].

\section{References:}

[1] S Vajda et al., Nature Materials 8 (2009), p. 213.

[2] WE Kaden et al., Science 326 (2009), p. 826.

[3] JY Liu, ACS Catalysis 7 (2017), p. 34.

[4] JY Liu, ChemCatChem 3 (2011), p. 934.

[5] JY Liu, Chinese Journal of Catalysis 38 (2017), p. 1460.

[6] RF Egerton, Micron 119 (2019), p. 72.

[7] This work was funded by NSF under CHE-1465057 and ACS PRF. We acknowledge the Eyring Materials Center and the John M. Cowley Center for High Resolution Electron Microscopy at Arizona State University.

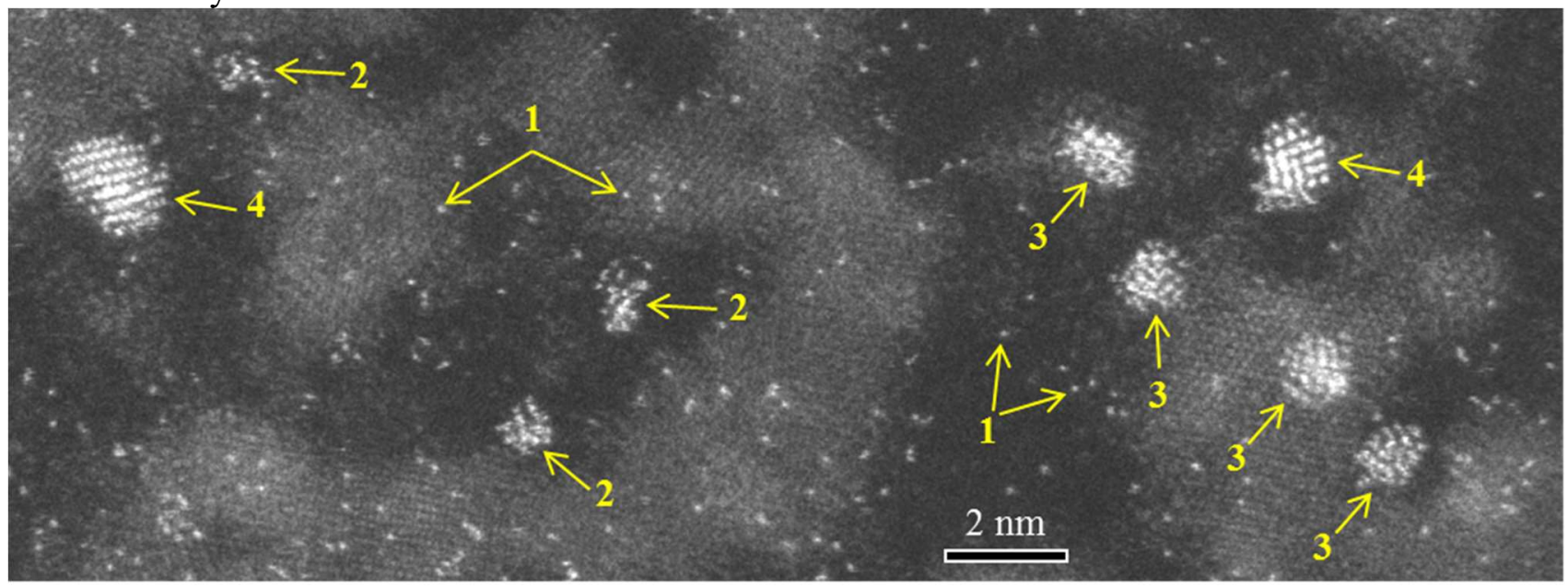

Figure 1. STEM-HAADF image of ultrathin $2 \mathrm{D} \mathrm{Co}_{3} \mathrm{O}_{4}$ nanosheet supported $\mathrm{Pt}$ atoms (indicated by \#1), subnano clusters (indicated by \#2), nanoclusters (indicated by \#3) and small nanoparticles (indicated by \#4).
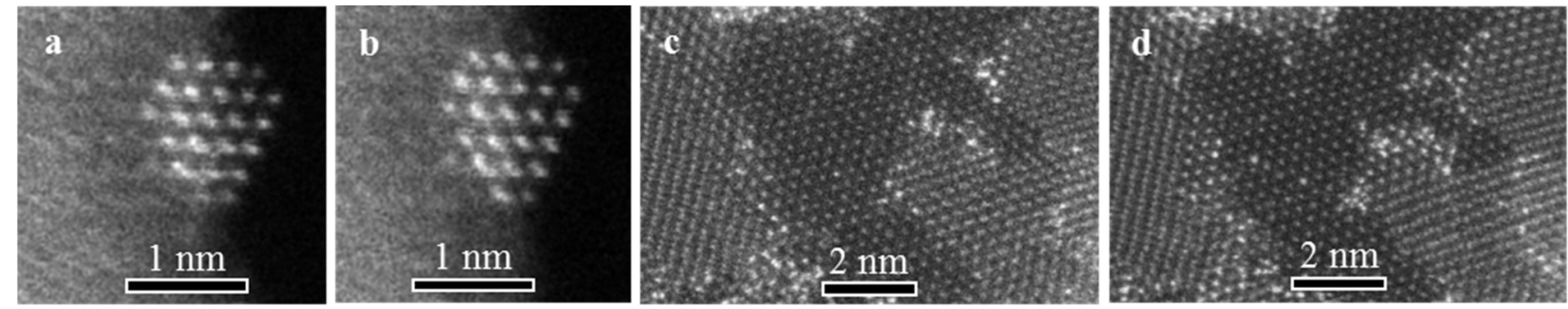

Figure 2. STEM-HAADF images of a subnano Ir cluster epitaxially anchored onto a ZnO nanowire (a) and after about $5 \mathrm{~min}$ of low-dose electron beam irradiation (b). STEM-HAADF images of Pt atoms and subnano clusters supported on $2 \mathrm{D} \mathrm{MoS}_{2}$ : first scan image (c) and second scan image (d), clearly demonstrating significant electronbeam induced effects on Pt atoms and clusters as well as monolayer edges of the $\mathrm{MoS}_{2}$ support. 